RESEARCH ARTICLE

\title{
Foot Care Education, Health-Seeking Behaviour and Disease Outcome in Patients with Diabetic Foot Ulcer: Results from the Multi-Centre Evaluation of Diabetic Foot Ulcer in Nigeria Study
}

\section{Innocent Okpe ${ }^{1^{*}}$, Ejiofor Ugwu ${ }^{2}$, Olufunmilayo Adeleye ${ }^{3}$, Ibrahim Gezawa ${ }^{4}$, Marcelina Enamino ${ }^{5}$ and Ignatius Ezeani ${ }^{6}$}

\author{
${ }^{1}$ Department of Medicine, Ahmadu Bello University, Kaduna, Nigeria \\ ${ }^{2}$ Department of Medicine, Enugu State University of Science and Technology, Enugu, Nigeria \\ ${ }^{3}$ Department of Medicine, Lagos State University, Lagos, Nigeria \\ ${ }^{4}$ Department of Medicine, Bayero University, Kano, Nigeria \\ ${ }^{5}$ Department of Medicine, Federal Medical Center, Nasarawa, Nigeria \\ ${ }^{6}$ Department of Medicine, Federal Medical Center, Abia, Nigeria
}

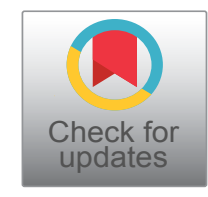

*Corresponding author: Innocent Okpe, Department of Medicine, Ahmadu Bello University, Zaria, Kaduna 810001, Nigeria, Tel: +234-8033-1464-31

\section{Abstract}

Background: Considerable advances have been made in the field of diabetic foot care over the past 25 years in developed countries. On the contrary, diabetes foot ulceration (DFU) still remains a common diabetes related complication of substantial public health importance in sub-Saharan Africa. Diabetic foot outcome in Nigeria is reportedly poor, with high amputation and mortality rates. Proper foot care education is known to impact positively on both DFU prevention and outcome in the developed societies. However, this relationship has not been well characterised in Nigeria.

Objective: To determine the relationship between diabetic foot care education, health-seeking behaviour and DFU outcome.

Methods: In this one-year observational Multi-Centre Evaluation of Diabetes Foot Ulcer in Nigeria (MEDFUN) study, subjects admitted for DFU in six tertiary hospitals were evaluated for demographic and diabetes related information, ulcer characteristics and treatment modality prior to hospitalisation. Prior participation in foot care education and knowledge of proper foot care were sought while relevant laboratory tests were performed. All patients benefitted from appropriate multidisciplinary care and were followed up until discharge or death. Outcome of interest were wound healing, lower extremity amputation (LEA) and mortality. Logistics regression was used to determine the impact of foot care education on DFU outcomes.
\end{abstract}

Results: Of the 336 patients enrolled for this study, $55.1 \%$ were male. The mean age of the participants was $55.9 \pm$ 12.5 years. Majority ( $96.1 \%$ ) had type 2 diabetes. Only a quarter $(25.9 \%)$ had prior foot care knowledge. Participants with foot care education were 5.762 times more likely to seek hospital treatment at onset of foot injury $(P<0.001)$, and were less likely to indulge in harmful practices such as native medication ( $P<0.001)$, visits to prayer houses $(P=0.161)$ and walking bare-footed $(P=0.009)$. Patients with prior foot care education also fared better on these treatment outcomes: LEA $(P=0.002)$, Wound infection $(P=$ $0.003)$ and Mortality $(P=0.445)$.

Conclusion: Diabetic foot care education has positive influence on health-seeking behaviour and diabetic foot clinical outcomes. There is therefore an urgent need to encourage physicians to periodically provide foot care.

\section{Keywords}

Foot care education, Health-seeking behaviour, Disease outcome, Diabetes foot ulcer, Nigeria

\section{Introduction}

Diabetic foot ulcers (DFU) are a major complication of diabetes seen in sub-Saharan Africa and the developing world where it remains a major healthcare problem of public health importance [1]. This

Citation: Okpe I, Ugwu E, Adeleye O, Gezawa I, Enamino M, et al. (2019) Foot Care Education, Health-Seeking Behaviour and Disease Outcome in Patients with Diabetic Foot Ulcer: Results from the Multi-Centre Evaluation of Diabetic Foot Ulcer in Nigeria Study. Int J Foot Ankle 3:038. doi. org/10.23937/2643-3885/1710038

Accepted: December 14, 2019; Published: December 16, 2019

Copyright: (C) 2019 Okpe I, et al. This is an open-access article distributed under the terms of the Creative Commons Attribution License, which permits unrestricted use, distribution, and reproduction in any medium, provided the original author and source are credited. 
common diabetes complication often result in considerable suffering to the affected with a tendency to recur frequently and is associated with high mortality as well as considerable healthcare costs [2].

The increasing prevalence of diabetes especially in sub-Saharan Africa and other developing countries, is likely to bring about a concomitant rise in its complications including diabetic foot ulcers [3]. Diabetic Foot Ulceration is a leading cause of hospital admissions among diabetes patients, and frequently result in lower extremity amputation (LEA) and consequently long-term disability and sometimes even death [3-5]. It has been estimated that up to a quarter of type 2 diabetes patients develop diabetic foot ulceration in their life time [3].

Diabetic foot complications constitute an increasing public health problem, not only in terms of the direct economic burden but also because of man-hour loss and the shear burden it places on our already weak health system $[1,6]$. For instance in Nigeria, the average cost of treatment of an uncomplicated DFU is estimated at between 1200 to 1,900 US Dollar and this cost continue to rise in the face of dwindling standard of living with the economic downturn in sub-Saharan Africa, including Nigeria due to poor governance, corruption and bad leadership [7-9]. Diabetic foot Ulceration has also earned notoriety for causing prolong hospital stay with some studies reporting a mean duration on admission as high as $59 \pm 41.9$ days (range 5 to 192 days) [10].

Diabetic foot ulceration is potentially preventable and the incidence can be curtailed through appropriate footcare education to all diabetes patients regardless of their perceived risk at the time of diagnosis $[11,12]$. The benefit of basic training on foot examination by self or a third party and the need to report any degree of injury to the foot as soon as it occurs to the appropriate hospital for necessary evaluation and prompt intervention cannot be overemphasized. Footcare education has long been advocated as an essential strategy of prevention of DFU, and has been widely practiced in the developed world [13-15]. The same cannot be said of our centres in Nigeria and the rest of the developing world, especially in Sub-Saharan Africa, where certified foot care educators and podiatrists are a rarity even in our referral centres [16-18]. The health system is often poorly organised, ill-equipped with poor referral system that provide incomplete coverage for the total diabetes population, majority of whom are at the understaffed primary healthcare level [18].

The current coverage of footcare education for people with diabetes in Nigeria and indeed the developing countries is not properly documented and the import of such absence of footcare knowledge amongst our diabetic population is unappreciated. We therefore sought to evaluate the availability of footcare education and its impact on health-seeking behaviour as well as DFU outcome.

\section{Methods}

The Multi-Centre Evaluation of Diabetes Foot Ulcer in Nigeria was an observational study of patients with type 1 or type 2 Diabetes Mellitus who were hospitalised for DFU in six tertiary healthcare institutions in Nigeria, between March 2016 and April 2017. These centres include Ahmadu Bello University Teaching Hospital Zaria (ABUTH) and Federal Medical Center Keffi (FMC Keffi) in North-Central Nigeria, Aminu Kano University Teaching Hospital Kano (AKTH) in North-Western Nigeria, Enugu State University Teaching Hospital (ESUTH) and Federal Medical Center Umuahia (FMC Umuahia) both in South- Eastern Nigeria and the Lagos State University Teaching Hospital Lagos (LASUTH) South-Western Nigeria. The Research and Ethics committee of ESUTH approved the study protocol while verbal informed consent was obtained from each patient prior to recruitment. Patients with diabetes other than type 1 and 2 DM were excluded.

Relevant socio-demographic and diabetes-related information as well as ulcer characteristics were documented. Patients were interviewed on knowledge of proper foot care practices and were required to indicate whether they had received foot care education prior to foot ulceration. History of development and progression of ulcer including mechanism of ulceration, site of ulcer, duration of ulcer and the first choice of treatment method were also assessed. Clinical wound infection was determined according to the International Working Group on Diabetes Foot (IWGDF) guideline by the presence of any two of the following: peri wound oedema, tenderness, differential warmth, wound exudates and foul smell [15]. Commonly known risk factors for DFU were also evaluated, including history of previous DFU, barefoot walking, improper foot wear, visual impairment, foot deformity, peripheral neuropathy- diagnosed by loss of pressure perception to Semmes-Weinstein $10 \mathrm{~g}$ monofilament test or diminished vibration sense using the $128 \mathrm{~Hz}$ tunning fork. Peripheral artery disease (PAD) was diagnosed when there was diminution or absence of dorsalis pedis and/or posterior tibial artery pulsations on manual palpation, ankle brachial index $(\mathrm{ABI})<0.9$ or significant arterial narrowing (> $50 \%$ ) on Doppler ultrasonography. The severity of ulcer was graded using two ulcer classification systems, namely, the Wagner's grading system and the University of Texas wound classification system $[19,20]$.

Relevant laboratory and imaging studies were performed for each subject including urine protein, full blood count, erythrocyte sedimentation rate (ESR), HbA1c, blood culture, ulcer specimen culture, lipid 
Table 1: Baseline demographic and clinical characteristics of the study population.

\begin{tabular}{|c|c|}
\hline \multicolumn{2}{|l|}{ Variable } \\
\hline Age (years) & $55.9 \pm 12.5$ \\
\hline$<45$ years & $48(14.3 \%)$ \\
\hline $45-64$ years & $200(59.5 \%)$ \\
\hline$\geq 65$ years & $88(26.2 \%)$ \\
\hline Male gender & $185(55.1 \%)$ \\
\hline Diabetes type (type 2) & $323(96.1 \%)$ \\
\hline Diabetes duration (years) & $8.5 \pm 5.7$ \\
\hline$\leq 10$ years & $250(74.4 \%)$ \\
\hline $11-20$ years & $79(23.5 \%)$ \\
\hline$>20$ years & $7(2.1 \%)$ \\
\hline Glycated hemoglobin $(\%)(n=296)$ & $9.6 \pm 1.9$ \\
\hline Had prior foot care knowledge & $87(25.9 \%)$ \\
\hline \multicolumn{2}{|l|}{ Type of Ulcer } \\
\hline Neuropathic & $125(37.2 \%)$ \\
\hline Ischemic & $42(12.5 \%)$ \\
\hline Neuro-ischemic & $135(40.2 \%)$ \\
\hline Unclassified & $34(10.1 \%)$ \\
\hline Duration of ulcer before admission (days) & $39(28-54)^{a}$ \\
\hline Previous history of foot ulcer & $96(28.6 \%)$ \\
\hline Advanced ulcer (Wagner grade $\geq 3$ ) & $266(79.2 \%)$ \\
\hline Presence of wound infection & $258(76.8 \%)$ \\
\hline \multicolumn{2}{|l|}{ Outcome } \\
\hline Suffered amputation & $119(35.4 \%)$ \\
\hline Left against medical advice & $35(10.1 \%)$ \\
\hline In-hospital mortality & $69(20.5 \%)$ \\
\hline Duration of hospitalization (days) & $50.8 \pm 28.0$ \\
\hline
\end{tabular}

Data are in number (percent) or mean $( \pm S D)$; a: median (interquartile range).

profile, plain radiograph of the foot and Doppler ultrasonography of both lower limbs. Co-morbid complications including hypertension, anaemia, shock, hyperglycaemic emergency, hypoglycaemia, stroke, kidney disease and cardiac failure were documented when present. Every patient received appropriate multi-disciplinary care and was followed up until discharge or death. Outcome variables of interest included ulcer healing, amputation, duration of hospitalisation and mortality. Other details of the MEDFUN protocol is as published elsewhere [21]. Here we present the sub-group analysis for the influence of diabetes foot care education on health-seeking behaviour of the subjects as well as on treatment outcome.

Data were collated in all the six participating centres and analysed using the Statistical Package for Social Sciences (IBM version 23.0; SPSS Inc., Chicago, IL, USA). Categorical variables were computed as numbers and percentages while continuous variables were presented as means and standard deviations or medians and interquartile ranges as appropriate. For this sub-analysis we tested for associations between demographic, clinical, treatment outcome variables and diabetes foot care education using logistics regression with statistical significance set at $P$-value of 0.05 .

\section{Results}

There was a combined total of 9,778 patients admitted on the medical wards of the participating institutions over the study period, of which 1,350 (13.8\%) were diabetes related, out of this number, 336 had DFU and were therefore enrolled for the study.

The basic demographic and clinical characteristics of the participants are shown in Table 1 . The study population (336) had a mean age of $55.9 \pm 12.5$ years $(55.1 \%$ male and $44.9 \%$ females). Type $2 \mathrm{DM}$ is more prevalent (96.1\%) and mean duration of diabetes for the entire study population was $8.5 \pm 5.7$ years. The mean $\mathrm{HbA1C}$ of the participants was $9.6 \pm 1.9 \%$. About one fourth (25.9\%) of the participants had prior knowledge of foot care. The predominant ulcer seen was neuro-ischemic $(40.2 \%)$, followed by neuropathic (37.2\%), ischemic $(12.5 \%)$ and the least was unclassified ulcer (10.1\%). - Most of the presentation of ulcer before admission ranges from 28 to 54 days and mean duration on admission was $50.8 \pm 28.0$ days. Overall, $28.6 \%$ of the patients had previous history of ulcer and $79.2 \%$ were advanced cases. Over one-third (35.4\%) suffered amputation. Other outcomes were in-hospital mortality $(20.5 \%)$ and discharge against medical advice (10.1\%).

Table 2 shows the association between demographic and clinical characteristics of patients and foot care education. There was no significant association between participant age $\{(\mathrm{OR}-1.129(95 \% \mathrm{Cl}=0.515-2.473)\}$, gen$\operatorname{der}\{(\mathrm{OR}-1.345 ; 95 \% \mathrm{Cl}=0.817-2.215\}$, type of diabetes $\{(\mathrm{OR}-1.867 ; 95 \% \mathrm{Cl}=0.594-5.870)\}$, duration of diabetes $\{(\mathrm{OR}-0.852 ; 95 \% \mathrm{Cl}=0.491-1.479)\}$ and foot care education ( $p$ - > 0.05 for all demographic characteristics).

Table 3 shows the association between foot care education and health seeking behavior of patients with diabetic foot. The patients who had foot care education were nearly 6 times more likely to visit hospital for their treatment $\{(\mathrm{OR}-5.762(95 \% \mathrm{Cl}=3.232$ 10.271) $p=0.001\}$. The results also show that subjects who had received foot care education were less likely to engage in self-medication as compared to those who had not received foot care education $\{(O R-$ $0.191(95 \% \mathrm{Cl}=0.113-3.325) \mathrm{p}=0.001\}$. Similarly, patients with foot care education were less likely to use native medication $\{(\mathrm{OR}-0.143(95 \% \mathrm{Cl}=0.052-0.343)$ $p=0.001\}$ and less likely to patronize prayer houses, however this relationship was not statistically significant $\{(\mathrm{OR}-0.459(95 \% \mathrm{Cl}=0.155-1.364) \mathrm{p}=0.161\}$.

Table 4 shows the impact of foot care education on ulcer characteristics and treatment outcome. The patients who suffered amputation were less likely to have received foot care education $\{(\mathrm{OR}-0.378(95 \% \mathrm{Cl}$ 
Table 2: Association between foot care knowledge and demographic and clinical variables.

\begin{tabular}{|c|c|c|c|c|c|}
\hline \multirow[t]{2}{*}{ Variable } & \multicolumn{2}{|c|}{ Foot care educated } & \multirow[t]{2}{*}{$P$ value } & \multirow[t]{2}{*}{ OR } & \multirow[t]{2}{*}{$95 \% \mathrm{Cl}$ for OR } \\
\hline & $\begin{array}{l}\text { Yes } \\
\text { n (\%) }\end{array}$ & $\begin{array}{l}\text { No } \\
\text { n (\%) }\end{array}$ & & & \\
\hline \multicolumn{6}{|c|}{ Age (years) } \\
\hline$<45$ & $13(27.1)$ & $35(72.9)$ & & & \\
\hline $45-64$ & $47(23.5)$ & $153(76.5)$ & 0.603 & 0.827 & $0.404-1.692$ \\
\hline$\geq 65$ & $26(29.5)$ & $62(70.5)$ & 0.762 & 1.129 & $0.515-2.473$ \\
\hline \multicolumn{6}{|l|}{ Gender } \\
\hline Male & $52(28.1)$ & $133(71.9)$ & 0.243 & 1.345 & $0.817-2.215$ \\
\hline Female & $34(22.5)$ & 117 (77.5) & & & \\
\hline \multicolumn{6}{|c|}{ Type of diabetes } \\
\hline Type 1 & $5(38.5)$ & $8(61.5)$ & 0.285 & 1.867 & $0.594-5.870$ \\
\hline Type 2 & $81(25.1)$ & $242(74.9)$ & & & \\
\hline \multicolumn{6}{|c|}{ Duration of diabetes (years) } \\
\hline$\leq 10$ & $62(24.8)$ & $188(75.2)$ & 0.569 & 0.852 & $0.491-1.479$ \\
\hline$>10$ & $24(27.9)$ & $62(72.1)$ & & & \\
\hline \multicolumn{6}{|c|}{ Glycemic control } \\
\hline Good & $5(6.6)$ & $10(4.5)$ & 0.488 & 1.479 & $0.489-4.473$ \\
\hline Poor & $71(93.4)$ & $210(95.5)$ & & & \\
\hline \multicolumn{6}{|c|}{ Neuropathy } \\
\hline Yes & $69(26.3)$ & $193(73.7)$ & 0.559 & 1.199 & $0.653-2.200$ \\
\hline No & $17(23.0)$ & $57(77.0)$ & & & \\
\hline \multicolumn{6}{|c|}{ Peripheral artery disease } \\
\hline Yes & $43(24.4)$ & $133(75.6)$ & 0.608 & 0.880 & $0.539-1.436$ \\
\hline No & $43(26.9)$ & $117(73.1)$ & & & \\
\hline
\end{tabular}

Table 3: Association between foot care education and health-seeking behavior of patients with diabetic foot ulcer.

\begin{tabular}{|c|c|c|c|c|c|}
\hline \multirow[t]{2}{*}{ Variable } & \multicolumn{2}{|c|}{ Foot care educated } & \multirow[t]{2}{*}{$P$ value } & \multirow[t]{2}{*}{ OR } & \multirow[t]{2}{*}{$95 \% \mathrm{Cl}$ for $\mathrm{OR}$} \\
\hline & $\begin{array}{l}\text { Yes } \\
\text { n (\%) }\end{array}$ & $\begin{array}{l}\text { No } \\
\text { n (\%) }\end{array}$ & & & \\
\hline \multicolumn{6}{|c|}{ Self medication } \\
\hline Yes & $32(37.2)$ & $189(75.6)$ & $<0.001$ & 0.191 & $0.113-0.323$ \\
\hline No & $54(62.8)$ & $61(24.4)$ & & & \\
\hline \multicolumn{6}{|c|}{ Native medication } \\
\hline Yes & $5(5.8)$ & $79(31.6)$ & $<0.001$ & 0.134 & $0.052-0.343$ \\
\hline No & $81(94.2)$ & $171(68.4)$ & & & \\
\hline \multicolumn{6}{|c|}{ Prayer house } \\
\hline Yes & $4(4.7)$ & $24(9.6)$ & 0.161 & 0.459 & $0.155-1.364$ \\
\hline No & $82(95.3)$ & $226(90.4)$ & & & \\
\hline \multicolumn{6}{|l|}{ Hospital } \\
\hline Yes & $68(79.1)$ & $99(39.6)$ & $<0.001$ & 5.762 & $3.232-10.271$ \\
\hline No & $18(20.9)$ & $151(60.4)$ & & & \\
\hline
\end{tabular}

$=0.205-0.698) \mathrm{p}=0.002\}$. Similarly, patients who had wound infection at presentation were less likely to have had foot care education $\{(\mathrm{OR}-0.444(95 \% \mathrm{Cl}=0.258-$ $0.764) p=0.003\}$ and those who suffered death were less likely to have had foot care education \{(OR- 0.779 (95\% Cl $=0.410-1.480)$. Similar things applied to those who engaged in barefoot walking $\{(\mathrm{OR}-0.512(95 \% \mathrm{Cl}$
$=0.309-0.849) p=0.009\}$ and those whose ulcers had lasted more than one month prior to presentation to hospital $\{(\mathrm{OR}-0.576(95 \% \mathrm{Cl}=0.343-0.967) \mathrm{p}=0.037\}$.

\section{Discussion}

Primary prevention by education remains a key concept even in the handling of diabetes complications, 
Table 4: Impact of foot care education on ulcer characteristics and outcomes.

\begin{tabular}{|c|c|c|c|c|c|}
\hline \multirow[t]{2}{*}{ Variable } & \multicolumn{2}{|c|}{ Foot care educated } & \multirow[t]{2}{*}{$P$ value } & \multirow[t]{2}{*}{ OR } & \multirow[t]{2}{*}{$95 \% \mathrm{Cl}$ for OR } \\
\hline & $\begin{array}{l}\text { Yes } \\
\text { n (\%) }\end{array}$ & $\begin{array}{l}\text { No } \\
\text { n (\%) }\end{array}$ & & & \\
\hline \multicolumn{6}{|c|}{ Engage in bare feet walking } \\
\hline Yes & $31(36.0)$ & $131(52.4)$ & 0.009 & 0.512 & $0.309-0.849$ \\
\hline No & $55(64.0)$ & $119(47.6)$ & & & \\
\hline \multicolumn{6}{|c|}{ Previous foot ulcer } \\
\hline Yes & $24(27.9)$ & $72(28.8)$ & 0.874 & 0.957 & $0.55-1.650$ \\
\hline No & $62(72.1)$ & $178(71.2)$ & & & \\
\hline \multicolumn{6}{|c|}{ Ulcer duration prior to admission } \\
\hline$\geq 1$ month & $53(61.6)$ & $184(73.6)$ & 0.037 & 0.576 & $0.343-0.967$ \\
\hline$<1$ month & $33(38.4)$ & $66(26.4)$ & & & \\
\hline \multicolumn{6}{|c|}{ Depth of ulcer } \\
\hline Superficial & $7(8.1)$ & $15(6.0)$ & 0.491 & 1.388 & $0.546-3.528$ \\
\hline Deep & $79(91.9)$ & $235(94.0)$ & & & \\
\hline \multicolumn{6}{|c|}{ Wound infection } \\
\hline Yes & $56(65.1)$ & $202(80.8)$ & 0.003 & 0.444 & $0.258-0.764$ \\
\hline No & $30(34.9)$ & $48(19.2)$ & & & \\
\hline \multicolumn{6}{|c|}{ Amputation } \\
\hline Yes & $16(21.1)$ & 93 (41.3) & 0.002 & 0.378 & $0.205-0.698$ \\
\hline No & $60(78.9)$ & $132(58.7)$ & & & \\
\hline \multicolumn{6}{|c|}{ Type of Amputation } \\
\hline Major & $15(78.9)$ & $75(75.0)$ & 0.714 & 1.250 & $0.379-4.118$ \\
\hline Minor & $4(21.1)$ & $25(25.0)$ & & & \\
\hline \multicolumn{6}{|l|}{ End point } \\
\hline Died & $15(19.7)$ & $54(24.0)$ & 0.445 & 0.779 & $0.410-1.480$ \\
\hline Survived & $61(80.3)$ & $171(76.0)$ & & & \\
\hline
\end{tabular}

including diabetic foot ulceration and where complications have already set-in, education on the appropriate ways to handle them remains a valuable means of guaranteeing a better outcome.

Our study demonstrated a very low level of foot care knowledge among the participants and it was therefore not surprising that DFU accounted for about a quarter of all diabetes related admissions during the period under study, a situation that is reflective of results from similar studies in our environment [22-24]. On the contrary, countries such as Germany and Belgium with a better organised healthcare system and well incorporated health education and training facilities, have far less diabetes admissions related to DFU, which are reportedly $<4.7 \%$ in Germany and $<10 \%$ in Belgium, even though there are variations within these countries $[25,26]$.

The mean age \pm SD of the participants in our study was $55 \pm 12.5$ years, indicating that those mostly affected were in their middle-ages and therefore belong to the most productive age bracket of the society. Thus the social, economic and developmental implications on a developing society such as ours are not far-fetched. This has been reported in similar studies around the world, where the most productive age groups are often the worst hit [16-18]. Our study also revealed that the males were more often the ones affected, probably because they are more active in out-door activities such as farming, mining and other high risk manual labours, as they are most of the time the breadwinners of their family.

Type 2 DM accounted for the majority (96.1\%) of the DFU cases seen in our study which is in keeping with the reported preponderance of type 2 DM worldwide [1]. The mean duration of diabetes of the participants was $8.5 \pm 5.7$ years, implying an early development of complications among our diabetes populations. This may probably be due to, but not limited to the problem of poor glycemic control of most of our participants-(mean $\mathrm{HbA1C}=9.6 \pm 1.9$ ) and that of late diagnosis of the disease in many of the patients in our locality [24-26]. Similar findings have been reported by Adeleye [10] and Anumah, et al. [9]. This is in sharp contrast to findings in Canada and Europe where most of the DFU complications occur after a longer duration of diagnosis of diabetes $[7,27]$.

Most of the DFU in this study presented late to hospi- 
tal with duration of onset of the ulcer before presentation ranging between 28 to 54 days. They present most often after exhausting the options available to them with little or no improvement, such as prayer houses, traditional healers and roadside drug stores or chemist etc. These initial choices are usually driven by ignorance and the belief that these places are often more accessible and affordable than orthodox hospitals; this attitude is promoted by the cultural and health belief system of the people. Another factor is the high cost of treatment in our hospitals where most payments is out-of-pocket as a result of a dysfunctional health insurance system whose coverage is less than $5 \%$ of the population [28]. This late presentation to hospital is reflective of other studies in this region $[9,10,12]$. Very often late presentation to hospital means more complications of the ulcer as $79.2 \%$ of our participants presented with advanced ulcers ( $\geq$ Wagner grade 3 ) and $76.8 \%$ presented with evidence of wound infection, resulting in longer duration of hospital stay and ultimately imparting negatively on outcome of treatment. This is comparable to the finding of Ogbera, et al. in their assessment of disease burden of DFU in a teaching hospital in Lagos [7].

In this study only $25.9 \%$ of participants had prior knowledge of foot care, a finding that is in keeping with other studies spanning over two decades from this part of the world $[7,9,10,17,18,22,27]$. This suggests that this pattern has remained consistent in communities in sub-Saharan Africa over time despite improvements in knowledge and technology in the field of diabetes foot care. This should be a source of serious concern because the diabetes epidemic projections for sub-Saharan Africa is quite gloomy; a whopping $156 \%$ increase in the next 2 decades, if we have to go by the current estimates by the International Diabetes Federation [1]. This gloomy picture of DFU statistics amongst other factors, may not be unconnected to the poor organisation of our clinics, which most often lack appropriate manpower staffing and training. Very few of our centres can boast of the appropriate level and complement of trained personal, such as diabetologists, diabetes nurses, diabetes educators, podiatrists, nutritionists. Even where they exist, they are most often overwhelmed by the shear number of patients demanding for attention [3]. This often means the patients cannot get a tailored and personalised education on diabetes footcare or even get to have their foot properly examined as often advocated as an effective means of reducing diabetes foot complications [29].

Our study did not show any significant association between participant age, gender, type of diabetes, duration of diabetes, glycemic control, type of ulcer and foot care education. Ordinarily it would be expected that there should be a positive correlation between foot care education and glycemic control, however this was not the case in our study. The prob- able explanation for the lack of association between glycemic control and foot care education in our study is that those with foot care knowledge may not necessarily practice what they have been taught. The implication is that, even amongst the few participants with foot care education, there is probably a gap between participant knowledge and self-practice which would require further researching. For instance, Rao $\mathrm{Li}$ and companions in their evaluation of the status of foot self-care knowledge and behaviour of nearly 6,000 type $2 \mathrm{DM}$ patients in China, found that although foot care knowledge was medium, based on a standard instrument they used for the study, there was no commensurate practice of foot self-care among the participants. They concluded that patients needed more guidance and regular training, including training to care givers before any tangible results can be achieved [30].

We also observed that the participants who had diabetes foot care knowledge were less likely to indulge in harmful practices, such as patronising native medication and visits to prayer houses and practiced less of self-medication and were nearly six times more likely to choose orthodox treatment as a first treatment option for their leg ulcer than their counterparts and these associations were statistically significant for each behaviour ( $P<0.001$ in each case). This goes to buttress the importance of foot care education as has been long espoused by several scholars [31,32]. Similarly our study also revealed that participants who were foot care educated were more likely to present to hospital early and were less likely to indulge in bare-foot walking which has been shown to be a risk factor in the development of DFU [2,22].

Our study also demonstrated some favourable outcome of treatment among those who had foot care education. These include; reduced chance of wound infection and the likelihood of LEA.

The cost of treating DFU in a developing country is huge. For instance, in low-income countries, the cost of treating a complicated DFU has been estimated to be the equivalent of 5.7 years of annual income of a family. This is financially catastrophic for the patients and their families [28]. This is so because of the low GDP and the fact that over $65 \%$ of the people live on less than a dollar per day. This huge economic cost of treatment of DFU makes it more expedient than ever, that appropriate action be taken to address this problem. Education and training is widely believed to be vital for stabilising the current epidemic of diabetes being witnessed in sub-Saharan Africa. In the same vain, we extrapolate that foot care education has the potential to play similar role in stabilising the menace of DFU being witnessed in sub-Saharan Africa. It follows therefore that foot care education programs should be seen as an investment in human capital 
which will not only save cost but will ultimately drive the much-desired development in sub-Saharan Africa.

A prospective longitudinal study over a longer period of follow-up with controls would have been preferable. Diabetic foot education was self-reported by the participants and therefore the exact content and nature of the foot care education received, remains a matter of conjecture. Despite this limitation the findings in our study provides valuable data for policy formulators and administrators to work on bringing the much-needed intervention in the field of diabetes foot care.

\section{Conclusion}

Foot care education has positive influences on health-seeking behaviour and DFU clinical outcomes. Hence it is important to encourage clinicians to periodically provide adequate foot care education to their patients.

\section{Consent Statement}

Written informed consent was obtained from the patients for publication of this research article. A copy of the written consent is available for review by the Editor-in-Chief of this journal.

\section{Declarations}

\section{Ethical approval}

The Ethics and Research committee of the Enugu State University Teaching Hospital, Enugu gave the ethical approval. The patients interviewed in this study did it voluntarily, and gave an informed consent.

\section{Source of funding}

None.

\section{Authors contribution}

IO and UE conceived of the study, carried out the sequence alignment and drafted the manuscript. OA, EI and IG participated in the sequence alignment, design of the study and helped to draft the manuscript. All authors read and approved the final manuscript.

\section{Acknowledgements}

We thank all the staff in our various institutions who contributed towards the article by making substantial contributions to conception and revision of manuscript for important intellectual content.

\section{Conflict of Interest}

We declare that the submitted work was carried out in the absence of any personal, professional or financial relationships that could potentially be construed as a conflict of interest.

\section{References}

1. International Diabetes Federation (2017) IDF Diabetes Atlas. ( $8^{\text {th }}$ edn), Brussels, Belgium.
2. Jeffcoate WJ, Vileikyte L, Boyko EJ, Armstrong DG, Boulton AJM (2018) Current challenges and opportunities in the prevention and management of diabetic foot ulcers. Diabetes Care 41: 645-652.

3. Rigato M, Pizzol D, Tiago A, Putoto G, Avogaro A, et al. (2018) Characteristics, prevalence, and outcome of diabetic foot ulcers in Africa. A systemic review and meta-analysis. Diabetes Res Clin Pract 142: 63-73.

4. Armstrong DG, Boulton AJM, Bus SA, (2017) Diabetic foot ulcers and their recurrence. N Engl J Med 376: 2367-2375.

5. Boulton AJ, Vileikyte L, Ragnarson-Tennvall G, Apelqvist J (2005) The global burden of diabetic foot disease. Lancet 366: 1719-1724.

6. Oguejiofor O, Odenigbo C, Onwukwe C (2014) Diabetes in Nigeria: Impact, Challenges, Future Directions. Endocrinol Metab Synd 3: 130.

7. Ogbera $A O$, Fasanmade $O$, Ohwovoriole AE, Adediran $O$ (2006) An assessment of the disease burden of foot ulcers in patients with diabetes mellitus attending a teaching hospital in Lagos, Nigeria. Int J Low Extrem Wounds 5: 244249.

8. Umar Mukhtar Danmusa, Iorliam Terhile, Idris Abdullahi Nasir, Auwal Alkasim Ahmad, Habiba Yahaya Muhammad (2016) Prevalence and healthcare costs associated with the management of diabetic foot ulcer in patients attending Ahmadu Bello University Teaching Hospital, Nigeria. Int J Health Sci (Qassim) 10: 219-228.

9. Anumah FO, Mshelia-Reng R, Abubakar A, Sough T, Asudo $F$, et al. (2017) Management outcome of diabetic foot ulcers in a teaching hospital in Abuja, Nigeria. J Diabetes Complicat 9: 15-20.

10. Adeleye JO (2005) Diabetic foot disease: The perspective of a Nigerian tertiary health care center. Practical Diabetes Int 22: 211-214.

11. Singh N, Armstrong DG, Lipsky BA (2005) Preventing foot ulcers in patients with diabetes. JAMA 293: 217-228.

12. Lepantalo M, Apelqvist J, Setacci C, Ricco JB, de Donato G, et al. (2011) Chapter V: Diabetic Foot. Eur J Vasc Endovasc Surg 42: S60-S74.

13. Pscherer S, Dippel FW, Lauterbach S, Kostev K (2012) Amputation rate and risk factors in type 2 patients with diabetic foot syndrome under real-life conditions in Germany. Prim Care Diabetes 6: 241-246.

14. Prompers L, Huijberts $M$, Apelqvist J, Jude E, Piaggesi $A$, et al. (2007) High prevalence of ischemia, infection and serious comorbidity in patients with diabetic foot disease in Europe. Baseline results from the Eurodiale Study. Diabetologia 50: 18-25.

15. Bakker K, Apelqvist J, Lipsky BA, Van Netten JJ (2016) The 2015 IWGDF guidance documents on prevention and management of foot problems in diabetes: Development of an evidence-based global consensus. Diabetes Metab Res Rev 32: 2-6.

16. Chinenye S, Young E (2011) State of Diabetes Care in Nigeria: A Review. The Nigerian Health Journal 11: 101-106.

17. Unachukwu C, Babatunde S, Ihekwaba AE (2007) Diabetes, hand and/or foot ulcers: A cross-sectional hospital-based study in Port Harcourt, Nigeria. Diabetes Res Clin Pract 75: 148-152.

18. Edo AE, Edo GO, Ezeani IU (2013) Risk factors, ulcer grade and management outcome of diabetic foot ulcers in a Tropical Tertiary care Hospital. Niger Med J 54: 59-63. 
19. Wagner FW Jr (1987) The diabetic foot. Orthopedics 10: 163-172.

20. Lavery LA, Armstrong DG, Harkless LB (1996) Classification of diabetic foot wounds. J Foot Ankle Surg 35: 528-531.

21. Ugwu E, Adeleye O, Gezawa I, Okpe I, Enamino M, et al. (2019) Burden of diabetic foot ulcer in Nigeria: Current evidence from the multicenter evaluation of diabetic foot ulcer in Nigeria. World J Diabetes 10: 200-211.

22. Oguejiofor OC, Oli JM, Odenigbo CU (2009) Evaluation of "care of the foot" as a risk factor for diabetic foot ulceration: The role of internal physicians. Niger J Clin Pract 12: 42-46.

23. Desalu OO, Salawu FK, Jimoh AK, Adekoya AO, Busari OA, et al. (2011) Diabetic foot care: Self-reported knowledge and practice among patients attending three tertiary hospital in Nigeria. Ghana Med J 45: 60-65.

24. Young EE, Anyim OB, Onyenekwe BM, Nwatu CB, Okafor $\mathrm{Cl}$, et al. (2016) Outcome of diabetic foot ulcer admissions at the medical wards of University of Nigeria Teaching Hospital Enugu, Nigeria. Int J Diabetes Dev Ctries 36: 220-227.

25. Zhang P, Lu J, Jing Y, Tang S, Zhu D, et al. (2017) Global epidemiology of diabetic foot ulceration: A systematic review and meta-analysis. Ann Med 49: 106-116.

26. Morbach S, Kersken J, Lobmann R, Nobels F, Doggen K, et al. (2016) The German and Belgian accreditation models for diabetic foot services. Diabetes Metab Res Rev 32: 318325.

27. Nyamu PN, Otieno CF, Amayo EO, Mc Ligeyo SO (2003) Risk factors and prevalence of diabetic foot ulcers at Kenyatta National Hospital, Nairobi. East Afr Med J 80: 36-43.

28. Ragnarson Tennvall G, Apelqvist J (2004) Health-economic consequences of diabetic foot lesions. Clin Infect Dis 39: S132-S139.

29. Dorresteijn JA, Valk GD (2012) Patient education for preventing diabetic foot ulceration. Diabetes Metab Res Rev 28: 101-106.

30. Meng Ren, Chuan Yang, Diao Zhu Lin, Hui Sheng Xiao, Li Fang Mai, et al. (2014) Effect of intensive nursing education on the prevention of diabetic foot ulceration among patients with high-risk diabetic foot: A Follow-Up Analysis. Diabetes Technol Ther 16: 576-581.

31. Mbanya JC, Motala AA, Sobngwi E, Assah FK, Enoru ST (2010) Diabetes in sub-Saharan Africa. Lancet 375: 22542266.

32. Brooke BS, Kraiss LW, Stone DH, Nolan B, De Martino RR, et al. (2014) Improving outcomes for diabetic patients undergoing revascularization for critical limb ischemia: Does the quality of outpatient diabetic care matter? Ann Vasc Surg 28: 1719-1728. 